\title{
Size of Sodium Dodecyl Sulfate Micelles in Aqueous Solutions as Studied by Positron Annihilation Lifetime Spectroscopy
}

\author{
G. Duplâtre* \\ Laboratoire de Chimie Nucléaire, Centre de Recherches Nucléaires, IN2P3/CNRS, B.P.28, \\ 67037 Strasbourg Cedex 2, France
}

\author{
M. F. Ferreira Marques \\ Instituto Superior de Engenharia, 3000 Coimbra, Portugal
}

\author{
M. da Graça Miguel \\ Departamento de Quimica da Universidade, 3000 Coimbra, Portugal
}

Received: February 29, 1996; In Final Form: June 3, $1996^{\otimes}$

\begin{abstract}
The micelles formed by sodium dodecyl sulfate (SDS) are studied in aqueous solutions at $294 \mathrm{~K}$ as a function of nominal SDS concentration $(C)$ using positron lifetime spectroscopy. In a four-component analysis of the data, it appears that the lifetime $\left(\tau_{3}\right)$ of the positronium triplet state $(o$-Ps) in the aqueous phase decreases significantly with increasing $C$. Preliminary results on sodium methanesulfonate solutions show that this compound does not result in any significant Ps lifetime quenching. Thus, the changes in the lifetime are attributed to the trapping of Ps from the aqueous to the organic micellar phase. On the basis of simple diffusion-controlled kinetics, the observed linearity of $1 / \tau_{3}$ with $C$ indicates that the ratio of the micelle core radius $\left(R_{\text {mic }}\right)$ to the aggregation number $\left(N_{\mathrm{ag}}\right)$ is about constant over the concentration range investigated, in agreement with information from other studies. Quantitatively, on the hypothesis of a constant value of $N_{\mathrm{ag}}$ $=64$, the use of the simple model, or of a more rigorous one, for Ps diffusion and trapping leads to $R_{\mathrm{mic}}=$ 1.50 and $1.19 \mathrm{~nm}$, respectively. Both values are lower than $1.84 \mathrm{~nm}$ as expected from other studies on the total SDS aggregate radius at $298 \mathrm{~K}$. However, taking account of the size of the polar sulfate groups and of some slight variation of $N_{\mathrm{ag}}$ with $C$, the most complete model leads to $R_{\mathrm{mic}}=1.75 \mathrm{~nm}$, in satisfactory agreement with the $1.84 \mathrm{~nm}$ value. Examination of the intensities of the two longest-lived components, related to $o$-Ps in the aqueous and micellar phases, shows that, as was the case for reverse micelles, most of the Ps formation occurs in the former. The aggregated SDS molecules display a very low inhibiting power toward Ps formation, with an inhibition constant of $0.01 \mathrm{M}^{-1}$, in agreement with the low ability of alkyl sulfates and sulfonates to scavenge electrons. The trapping of Ps in the micelles is confirmed by the continuous increase with $C$ of the intensity of the longest-lived component, related to $o$-Ps in the organic phase.
\end{abstract}

\section{Introduction}

In a recent paper, positron annihilation lifetime spectroscopy (LS) was applied to the study of a reverse micellar system, AOT/ water microemulsions in isooctane. ${ }^{1}$ The probe used was the long-lived triplet state ( $o$-Ps) of positronium (Ps), the bound state of a positron with an electron, whose lifetime and relative abundance, or intensity, both depend on the physicochemical properties of the medium. As expected, the LS spectra analysis showed the existence of two long-lived components physically corresponding to $o$-Ps present in either the organic $\left(o-\mathrm{Ps}{ }^{\text {org }}\right)$ or the aqueous micellar $\left(o-\mathrm{Ps}^{\mathrm{aq}}\right)$ phases. The main findings were as follows. (i) Although its volume fraction is limited to a few percent, the aqueous core of the micelles is much more efficient than the organic phase for slowing the energetic positrons in such a way that a large fraction of Ps is formed in the micelles. (ii) Once formed, Ps can diffuse out from the micelle cores to the organic phase. This was established on the observation that the $o-\mathrm{Ps}^{\mathrm{aq}}$ lifetime is substantially reduced as compared to pure water. For the first time, by setting rigorous diffusion equations, quantitative information could be derived on the micellar core radius as a function of various parameters: AOT/water ratio, AOT concentration, and temperature.

\footnotetext{
${ }^{\otimes}$ Abstract published in Advance ACS Abstracts, August 15, 1996.
}

Although some normal micellar systems have been studied by $\mathrm{LS},{ }^{2-7}$ no such noteworthy phenomenon as the $o$-Ps lifetime decrease has been reported. In view of our previous results, it seemed therefore promising to reinvestigate one of these systems, sodium dodecyl sulfate (SDS) in aqueous solutions, to assess the presence of two Ps states on the basis of a fourcomponent analysis of the LS spectra instead of the previous three-component analyses.

Preliminary experiments were carried out on aqueous solutions of sodium methanesulfonate, which does not form micelles and should give reliable information on the effect on Ps of the isolated SDS molecules.

\section{Experimental Section}

The chemicals, sodium methanesulfonate and dodecyl sulfate, were AR grade commercial compounds, used as received.

The positron source consisted of $4 \times 10^{5} \mathrm{~Bq}^{22} \mathrm{Na}$ diffused in a thin sodium glass film, sustained by an all-glass holder, yielding a source correction of $8 \%$. The source was immersed in the solution to be studied, inside a Pyrex tube previously degassed through the usual freeze-thaw technique, then sealed.

The LS spectrometer, based on NE111 scintillators, had a time resolution of $290 \mathrm{ps}$. To avoid any distortion in the spectra due to the non-Poisson distribution of the counts in each channel, 


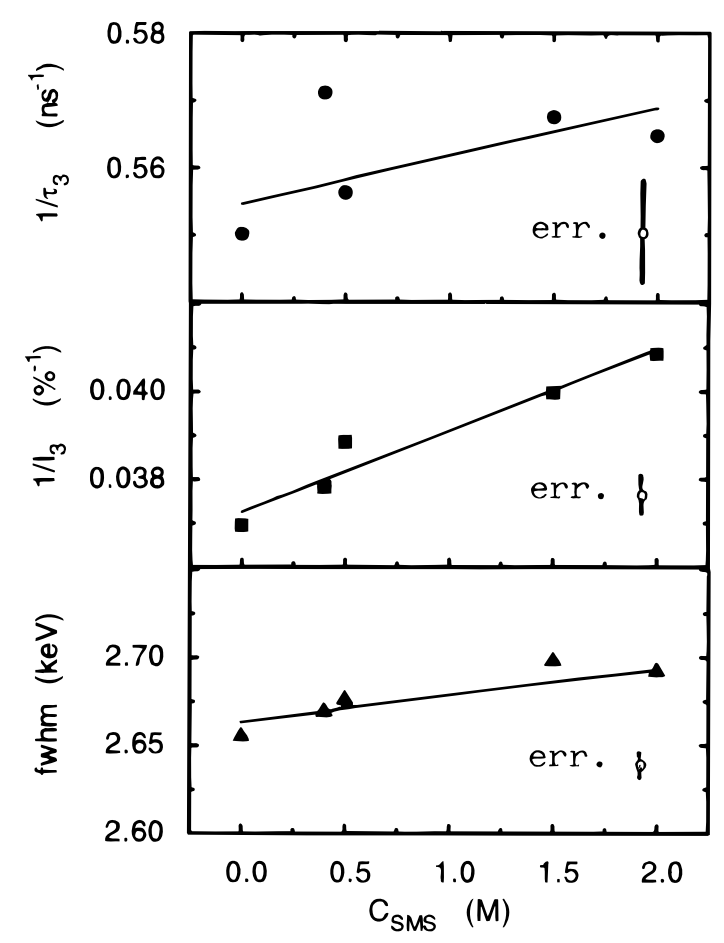

Figure 1. Variation of the LS and DBARL parameters for sodium methanesulfonate in aqueous solutions as a function of concentration, $C_{\text {SMS }}(\mathrm{M}):(\mathbf{O}) o$-Ps decay rate constant, $1 / \tau_{3}\left(\mathrm{~ns}^{-1}\right)$; $(\mathbf{\square})$ inverse of $o$-Ps intensity, 1/I3 $\left(\%^{-1}\right) ;(\boldsymbol{\Lambda})$ fwhm $(\mathrm{keV})$.

the integrated counts were kept in the range $(5-8) \times 10^{5}$. The temperature was maintained at $(294 \pm 0.4) \mathrm{K}$. In threecomponent analyses of the spectra, the quality of fit was progressively deteriorated as the SDS concentration increased: above about $0.2 \mathrm{M}$, the spectra could no longer be satisfactorily analyzed, giving $\chi^{2}>1.2$. In a next step, the spectra were therefore analyzed in terms of four components, physically ascribable to the short-lived singlet states of Ps ( $p$-Ps), free positrons and triplet states in either the aqueous or micellar organic phases. The lifetimes, $\tau_{i}$, and decay rate constants, $\lambda_{i}$ $=1 / \tau_{i}$, as well as the intensities, $I_{i}$, will be denoted by subscripts $i=1,2,3$, and 4 , accordingly. The LS parameters in the neat phases will be further denoted by superscripts "aq" and "mic". For the three-component analysis of the sodium methanesulfonate samples, the experimental errors were at about 0.025 ns and $0.2 \%$ for $\tau_{3}$ and $I_{3}$, respectively.

The Doppler broadening of the annihilation radiation line shape (DBARL) experiments were carried out using an hyperpure $\mathrm{Ge}$ detector with a resolution of $1.40 \mathrm{keV}$ at the annihilation peak. The results will be presented in terms of the fwhm of this peak. ${ }^{8}$ The experimental error of fwhm was within 0.01 $\mathrm{keV}$.

\section{Results and Discussion}

A. Sodium methanesulfonate (SMS). As shown in Figure 1 , the variations of the LS parameters for $o$-Ps, $\tau_{3}$, and $I_{3}$, are very smooth. As found for numerous solutes, ${ }^{9,10}$ both $1 / I_{3}$ and $1 / \tau_{3}$ vary linearly with the concentration of solute, $C$, allowing us to define a Ps inhibition constant $(k)$ and a Ps lifetime quenching rate constant $\left(k^{\prime}\right)$ according to

$$
\begin{gathered}
I_{3}=I_{3}^{\mathrm{aq}} /(1+k C) \\
1 / \tau_{3}=1 / \tau_{3}^{\mathrm{aq}}+k^{\prime} C
\end{gathered}
$$

Fitting the data using eqs 1 and 2 leads to $k=(0.050 \pm 0.005)$
$\mathrm{M}^{-1}$ and $k^{\prime}=(0.007 \pm 0.002) \mathrm{M}^{-1} \mathrm{~ns}^{-1}$, respectively (solid lines in Figure 1). Both values show that SMS has little effect on either Ps formation or lifetime.

As is the case for other rather chemically inert solutes, ${ }^{9,10}$ the very low value of $k^{\prime}$ indicates that the decrease in $o$-Ps lifetime as $C$ increases is most probably not due to a chemical reaction of Ps with SMS but merely arises from the increase in the electronic density of the medium, resulting in an enhancement of the pick-off process by which $o$-Ps annihilates.

Regarding the $o$-Ps intensity, the spur model proposes that Ps is formed by the reaction of $\mathrm{e}^{+}$with one of those quasi-free electrons created through ionization of the medium at the end of the positron track. This hypothesis has been strongly supported by the correlations found between the inhibition constants of a large variety of solutes and their reaction rate constant with solvated electrons or, even better, with their constant for scavenging the precursors of the solvated electron. ${ }^{9,10}$ Alkyl sulfates and sulfonates, including SDS, are known to be relatively unreactive toward solvated electrons, with reaction rate constants ${ }^{11-13}$ below $10^{7} \mathrm{M}^{-1} \mathrm{~s}^{-1}$, in agreement with the low value for $k$.

In DBARL experiments, the weak inhibition is confirmed (Figure 1): qualitatively, the smooth increase in fwhm with SMS concentration arises from the disappearance of the two narrowest components, $p$-Ps and $o$-Ps, upon inhibition. Quantitatively, the data are very well described without any free parameter by calculating the DBARL distribution in terms of a sum of Gaussian components ${ }^{8}$ with intrinsic fwhms at $\Gamma_{1}=0.88 \mathrm{keV}$, $\Gamma_{2}=2.67 \mathrm{keV}$, and $\Gamma_{3}=2.40 \mathrm{keV}$ as previously determined for $p$-Ps, free $\mathrm{e}^{+}$, and $o$-Ps, respectively (subscripts 1,2 , and 3 are kept in the order of increasing lifetimes). ${ }^{14}$ The very good agreement between the calculated (solid line in Figure 1) and experimental plots corroborates an inhibition through electron capture by SMS: a reaction with the positron to yield a bound state would have required an additional unknown parameter descriptive of this bound state for calculation of the fwhm variation.

B. Sodium Dodecyl Sulfate (SDS) in Aqueous Solutions. In all-free four-component analyses of the spectra, the lifetime of the longest-lived component, related to $o$-Ps decay in the organic core of the micelles, is poorly defined for SDS concentrations below $0.1 \mathrm{M}$. This low reproducibility is due to the weak associated intensity, $I_{4}$, which is less than $5 \%$ in this concentration range. Above $0.1 \mathrm{M}$, the LS spectra become progressively better defined and reproducible. However, the average error remains higher than usual with an average value of $\tau_{4}=(3.7 \pm 0.1) \mathrm{ns}$ for the range $0.125-0.5 \mathrm{M}$. This value is somewhat higher than $3.43 \mathrm{~ns}$ found for $n$-dodecane in a previous work. ${ }^{15}$ Taking account of the presence of the anionic sulfate heads of the micelles, a value lower than 3.43 ns would be more expected owing to the increase in the electron density as compared with the value for pure $n$-dodecane. Therefore, for the sake of coherence of the LS parameters the data were also analyzed with $\tau_{4}$ fixed at the above average value.

By contrast, the lifetime of the second longest-lived component, related to $o$-Ps decay in pure water, is well defined: in the concentration range $0-0.5 \mathrm{M}$, the experimental error on $\tau_{3}$ varies from $0.020 \mathrm{~ns}$ to about $0.045 \mathrm{~ns}$ and from $0.025 \mathrm{~ns}$ to $0.060 \mathrm{~ns}$, whether $\tau_{4}$ is fixed or not, respectively. With $\tau_{4}$ set at $3.7 \mathrm{~ns}$, the experimental errors on the intensities $I_{3}$ and $I_{4}$ were both at about $0.3 \%$.

As shown in Figure 2, the most striking feature is the constant decrease of $\tau_{3}$ as $C$ increases, leading to an apparent linear variation of $1 / \tau_{3}$. This phenomenon was not noted in the previous studies on SDS based on LS measurements. ${ }^{5-7}$ The 


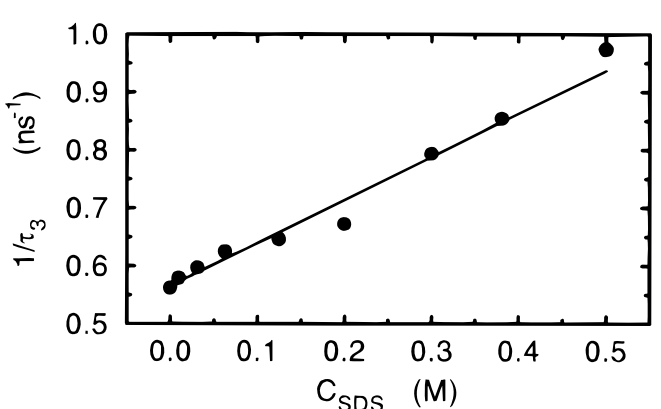

Figure 2. Variation of the decay rate constant, $1 / \tau_{3}\left(\mathrm{~ns}^{-1}\right)$, of the second longest-lived component, corresponding to $o$-Ps in water, in SDS aqueous solution as a function of concentration, $C_{\mathrm{SDS}}(\mathrm{M})$. For the definition of the solid line, see text.

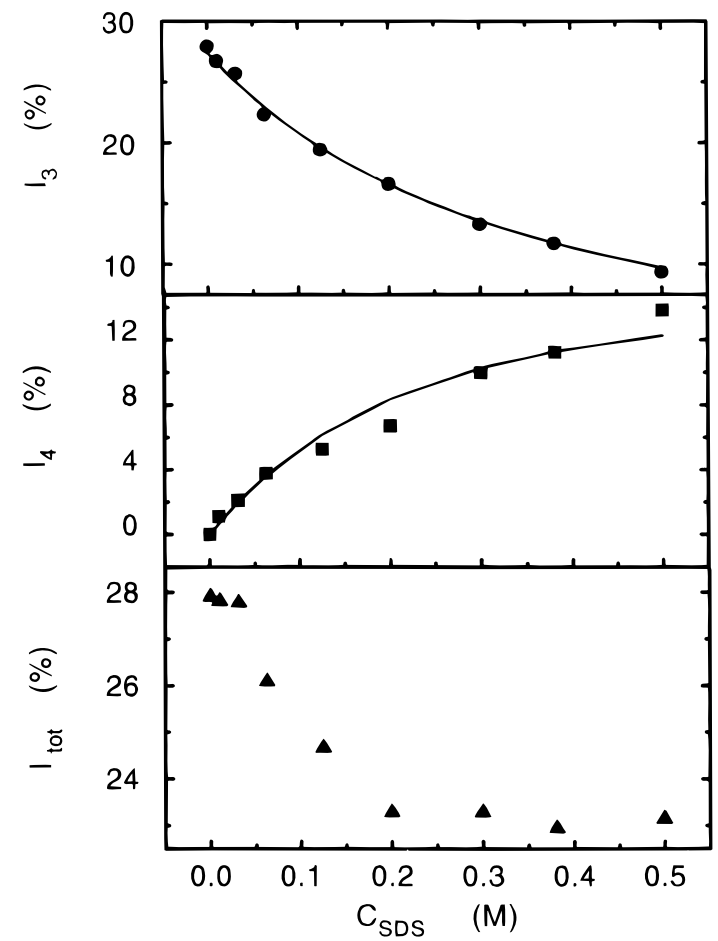

Figure 3. Variation of the intensitites of the two longest-lived components in SDS aqueous solutions: (-) second longest-lived component (o-Ps in water), $I_{3}(\%)$; $(\mathbf{\square})$ longest-lived component $(o$-Ps in micelles), $I_{4}(\%)$; $(\boldsymbol{\Delta})$ total intensity $\left(I_{3}+I_{4}\right)$. For definition of the solid lines, see text.

intensities, $I_{3}$ and $I_{4}$, respectively decrease and increase with increasing $C$ (Figure 3). However, as was the case for the reverse micelles, ${ }^{1}$ the total intensity, $I_{\text {tot }}=I_{3}+I_{4}$, decreases very smoothly with $C$ (Figure 3 ). In the following, the lifetime and intensity variations are analyzed and discussed successively.

C. $o$-Ps ${ }^{\text {aq }}$ Lifetime in SDS Solutions. As shown in Figure 1 , it is not likely that such a molecule as SDS would present any significant reactivity toward Ps: only those solutes having a very strong reactivity (in water, strong oxidizers) ${ }^{9}$ are likely to quench $\tau_{3}$ in such a way as shown in Figure 2. Furthermore, if Ps were to react chemically with the micelles, a significant increase in $I_{3}$ should be observed in the absence of any strong inhibition ${ }^{9}$ and the lifetime of the product of the reaction is not likely to be at about $3.7 \mathrm{~ns}$ as is experimentally observed. Therefore, the decrease in $\tau_{3}$ arises from the diffusion of Ps from the aqueous phase to the organic core of the micelles in a similar way to that found for the reverse micelles. ${ }^{1}$ The corresponding kinetic scheme is thus as follows:

$$
2 \gamma \stackrel{\lambda_{\mathrm{aq}}^{3}}{\longleftrightarrow} o-\mathrm{Ps}^{\mathrm{aq}} \stackrel{k_{\mathrm{dif}}}{\longrightarrow} o-\mathrm{Ps}^{\mathrm{mic}} \stackrel{\lambda_{4}^{\text {mic }}}{\longrightarrow} 2 \gamma
$$

Quantitatively, the simplest approach to this scheme is by using the approximate expression for the diffusion-controlled reaction rate constant with a spherical solute (subscripts 4 and 5 for $k_{\mathrm{dif}}$ in what follows make reference to the corresponding equations):

$$
k_{\mathrm{dif} 4}=4 \pi D_{\mathrm{Ps}} R_{\mathrm{mic}} N_{\mathrm{Av}} / 1000
$$

where $D_{\mathrm{Ps}}$ is the Ps diffusion coefficient $\left(D_{\mathrm{Ps}}=4.15 \mathrm{~nm}^{2} \mathrm{~ns}^{-1}\right.$ at $294 \mathrm{~K}),{ }^{1} R_{\mathrm{mic}}$ is the micelle radius, and $N_{\mathrm{Av}}$ is the Avogadro number. An alternative equation that is commonly used in the field is as follows: ${ }^{16,17}$

$$
k_{\mathrm{dif5}}=2 N_{\mathrm{Av}} k_{\mathrm{B}} T\left(2+R_{\mathrm{Ps}} / R_{\mathrm{mic}}+R_{\mathrm{mic}} / R_{\mathrm{Ps}}\right) /(3000 \eta)
$$

where $k_{\mathrm{B}}$ is the Boltzmann constant, $T$ is the absolute temperature, $R_{\mathrm{Ps}}=0.053 \mathrm{~nm}$ is the Ps radius, and $\eta$ is the viscosity of the medium. In either case, the time differential equations for $o$-Ps are given by

$$
\begin{gathered}
\mathrm{d}\left(o-\mathrm{Ps}^{\mathrm{aq}}\right) / \mathrm{d} t=-\left(\lambda_{\mathrm{aq}}^{3}+k_{\mathrm{dif}} C_{\mathrm{mic}}\right) o-\mathrm{Ps}^{\mathrm{aq}} \\
\mathrm{d}\left(o-\mathrm{Ps}^{\mathrm{mic}}\right) / \mathrm{d} t=-\lambda_{4}^{\mathrm{mic}} o-\mathrm{Ps}{ }^{\mathrm{mic}}+k_{\mathrm{dif}} C_{\mathrm{mic}} o-\mathrm{Ps}^{\mathrm{aq}}
\end{gathered}
$$

where $C_{\text {mic }}$ is the micelle concentration given by the ratio of the nominal SDS concentration, $C$, to the average aggregation number, $N_{\text {ag: }}$ :

$$
C_{\text {mic }}=C / N_{\mathrm{ag}}
$$

On the basis of eqs 6 and 7, the second longest lifetime is therefore

$$
1 / \tau_{3}=1 / \tau_{3}^{\mathrm{aq}}+k_{\mathrm{dif}} C / N_{\mathrm{ag}}
$$

From the good linearity of the experimental variation of $1 / \tau_{3}$ with $C$, it is thus expected from eqs 4 and 9 that the ratio $R_{\text {mic }} /$ $N_{\text {ag }}$ be essentially constant over the concentration range studied. The same is true for eq 5 , provided that $R_{\text {mic }} \gg R_{\text {Ps. }}$.

The approximations implicit in eqs 4 and 5 can be circumvented by using a more rigorous approach to the problem of Ps diffusion and trapping in a sphere. The relevant equations have been published previously ${ }^{18,19}$ with the aim of describing the recombination reaction of pairs of defects in irradiated solids. Supposing that Ps can enter the micellar spheres on each encounter, the time differential equation for $o$-Ps ${ }^{\mathrm{aq}}$ is given by

$$
\begin{aligned}
& -\mathrm{d}\left(o-\mathrm{Ps}^{\mathrm{aq}}\right) / \mathrm{d} t= \\
& \left\{k_{\mathrm{dif} 4} C_{\mathrm{mic}}\left[1+R_{\mathrm{mic}} /\left(\pi D_{\mathrm{Ps}} t\right)^{1 / 2}\right]+\lambda_{3}^{\mathrm{aq}}\right\} o-\mathrm{Ps}^{\mathrm{aq}}
\end{aligned}
$$

where $k_{\text {dif }}$ is given by eq 4 . Integrating eq 10 gives

$$
\begin{aligned}
& o-\mathrm{Ps}^{\mathrm{aq}}= \\
& \quad A \exp \left\{-k_{\mathrm{dif} 4} C_{\mathrm{mic}}\left[t+2 R_{\mathrm{mic}} t^{1 / 2} /\left(\pi D_{\mathrm{Ps}}\right)^{1 / 2}\right]-\lambda_{3}^{\mathrm{aq}} t\right\}
\end{aligned}
$$

which is conveniently rearranged as

$$
o-\mathrm{Ps}^{\mathrm{aq}}=A \exp \left(-\alpha t-\beta t^{1 / 2}\right)
$$

with $\alpha=k_{\mathrm{dif} 4} C_{\mathrm{mic}}+\lambda_{3}^{\mathrm{aq}}$ and $\beta=2 k_{\mathrm{dif} 4} C_{\mathrm{mic}} R_{\mathrm{mic}} /\left(\pi D_{\mathrm{Ps}}\right)^{1 / 2}$. A striking feature of eq 12 is that, on rigorous physical grounds, the diffusion of Ps to the micelle droplets introduces a term in $t^{1 / 2}$ in the exponential, while the fitting of the LS spectra is made on the basis of decaying exponential functions whose arguments are linear with $t$ in the form $B \lambda \exp (-\lambda t)$. Mathematically then, the LS fitting programs aim at minimizing the $\chi^{2}$ defined through the sum, over all channels, of the squares of the differences between these two types of functions, in relation to the LS 
parameters, intensity, and decay rate constant. For the second longest-lived component, the latter is thus obtained through

$$
\mathrm{d} / \mathrm{d} \lambda_{3} \int_{0}^{\infty}[F(t)-G(t)]^{2} / F(t) \mathrm{d} t=0
$$

where $F(t)$ is given by eq 12 and $G(t)=B \lambda_{3} \exp \left(-\lambda_{3} t\right)$. Note that the exact definition of the pre-exponential terms $A$ and $B$ in the preceding equations is not relevant here, since only the decay rate constant is examined; the denominator in eq 13 represents the square of the statistical error. The effectiveness of eq 13 is easily verified in a simulation experiment by fitting a calculated spectrum of the form of eq 12 by the usual LS fitting programs.

Equation 13 can be simplified to

$$
\mathrm{d} / \mathrm{d} \lambda_{3} \int_{0}^{\infty} \lambda_{3}^{2} \exp \left[-\left(2 \lambda_{3}-\alpha\right) t+\beta t^{1 / 2}\right] \mathrm{d} t=0
$$

which gives the following equation for $\lambda_{3}$ :

$$
2\left(\lambda_{3}-\alpha\right)\left[1+\beta H\left(\lambda_{3}\right)\right]+\beta \lambda_{3}\left(2 \lambda_{3}-\alpha\right) \mathrm{d} H\left(\lambda_{3}\right) / \mathrm{d} \lambda_{3}=
$$

where $H\left(\lambda_{3}\right)=\int_{0}^{\infty} \exp \left[-\left(2 \lambda_{3}-\alpha\right) t^{2}+\beta t\right] \mathrm{d} t$. Equation 15 is conveniently solved by an iteration procedure, with $H\left(\lambda_{3}\right)$ and $\mathrm{d} H\left(\lambda_{3}\right) / \mathrm{d} \lambda_{3}$ developed in terms of rapidly converging series.

Quantitatively, fitting the data on the basis of eqs 9 or 15 involves two parameters: $R_{\text {mic }}$ and $N_{\text {ag }}$. For SDS at $298 \mathrm{~K}, N_{\mathrm{ag}}$ is considered to be reasonably constant above the critical micellar concentration, and a value of $N_{\mathrm{ag}}=64$ appears to be well established by various techniques. ${ }^{20-22}$ Similarly, the apparent molar volume of aqueous SDS at $298 \mathrm{~K}$ does not vary markedly $^{22,23}$ and the data show that, for $N_{\mathrm{ag}}=64$, the SDS micelle radius should only change ${ }^{22}$ from 1.82 to $1.85 \mathrm{~nm}$ in the range $0.01-0.5 \mathrm{M}$, with an average value at $1.84 \mathrm{~nm}$.

The apparent slope of the plot in Figure 2 is $k_{\mathrm{dif}} / N_{\mathrm{ag}}=0.732$ $\mathrm{M}^{-1} \mathrm{~ns}^{-1}$ with a standard deviation of $0.029 \mathrm{~ns}$, which compares well with the experimental errors (solid line in Figure 2). With $N_{\text {ag }}=64$, this leads to $R_{\text {mic }}=1.51$ and $1.41 \mathrm{~nm}$ when using eqs 4 and 5 for $k_{\text {dif }}$, respectively, while eq 15 leads to a similar fit of the data with $R_{\text {mic }}=1.19 \mathrm{~nm}$.

Data on the mean thermodynamical radius of SDS micelles in the presence of $\mathrm{NaCl}$ show that it decreases significantly with increasing temperature for $\mathrm{NaCl}$ concentrations above $0.4 \mathrm{M}$ and tends to be somewhat constant when $\mathrm{NaCl}$ is progressively removed. ${ }^{24}$ Therefore, no significant effect of the temperature between $298 \mathrm{~K}(1.84 \mathrm{~nm})$ and $294 \mathrm{~K}(1.19 \mathrm{~nm})$ is expected. Similarly, the relative purity of the chemical should not have any important influence, since previous work shows that for various high-purity batches of SDS, the difference in the radius at $298 \mathrm{~K}$, although well distinguished, is only from 1.84 to 1.85 $\mathrm{nm}$ on the basis of $N_{\mathrm{ag}}=64 .^{23}$

The value of $1.84 \mathrm{~nm}$ is deduced from the measured molar volume of whole SDS molecules. In our case, as shown later by considering the inhibition effect of the sulfate groups, which applies exclusively to the aqueous phase, a significant change in the $o$-Ps lifetime most probably occurs only as this particle penetrates the organic phase built up by the dodecane molecules. On this basis, the LS data would deliver information on the organic micellar core radius so that about $0.5 \mathrm{~nm}$, representative of the sulfate heads diameter, should be added. Taking $R_{\text {mic }}=$ $1.19 \mathrm{~nm}$ as the most reliable value to express the LS data, this yields a total radius of $1.69 \mathrm{~nm}$, which is in better agreement with the value found using other techniques.

On the other hand, the value of $N_{\mathrm{ag}}=64$ is only an average and previous results show that $N_{\text {ag }}$ varies somewhat with SDS concentration $(C)$ in a roughly linear way, as ${ }^{25,26}$

$$
N_{\mathrm{ag}}=17.47 C+61.71
$$

Using eq 15 with $N_{\mathrm{ag}}$ defined as above leads to an average radius $R_{\text {mic }}=1.25 \mathrm{~nm}$, which, taking account of the sulfate groups diameter, gives a total SDS micelle radius of $1.75 \mathrm{~nm}$, in good agreement with $1.84 \mathrm{~nm}$ deduced from other techniques on the hypothesis that $N_{\text {ag }}$ is constant at 64 .

Thermodynamically, it has been pointed out that for the AOT/ water microemulsions ${ }^{1}$ the process of Ps trapping in the organic phase should be unfavorable: the bubble state in which Ps is supposed to exist in liquids would represent a deeper trap, by about $0.4 \mathrm{eV}$, in water as compared to alkanes. Strangely enough too, the rate constants for Ps diffusion-controlled reactions with a very large variety of solutes are very well calculated, with no fitting parameter, on the basis of equations similar to eqs 4 and 5. ${ }^{17,27-29}$ These expressions are based on the Smoluchowski equation, which involves the diffusion coefficient of the reactive species, and the reaction rate constants are calculated by using the Ps diffusion coefficient. For a rigid bubble, the bubble radius in water can be estimated to $0.26 \mathrm{~nm}$ from the $o$-Ps lifetime and the water surface tension (72.59 dyn $\mathrm{cm}^{-1}$ at $\left.294 \mathrm{~K}\right){ }^{29}$ Since the diffusion coefficient of the reactive species is inversely proportional to its radius, on the basis of the Stokes-Einstein equation implicit in eq 5, this implies that the Ps bubble, considered as the diffusing reactant, is some 5 times less reactive than Ps. Consequently, fitting the data with eqs 4 or 5 leads to values of $R_{\text {mic }}$ that are by far too high, at about $5.4 \mathrm{~nm}$, while eq 15 leads to some $3.4 \mathrm{~nm}$. This inconsistency between the bubble concept, which is supported by a number of experimental facts, ${ }^{29}$ and the quantitative evaluation of the Ps reaction rate constants seems difficult to overcome at the moment. Perhaps a hint for the resolution of this problem may be found by considering that the bubble is dynamically created as Ps moves along in the liquid so that the movement of the bound system can be treated as that of a free Ps atom in the relevant diffusion equations.

D. o-Ps Intensities in SDS Aqueous Solutions. Quantitatively, the intensities related to the two longest-lived components can be conveniently treated on the basis of reaction 3 . On this basis, the relevant equations for $I_{3}$ and $I_{4}$ are

$$
\begin{gathered}
I_{3}=I_{3}^{\mathrm{aq}} F(C)\left(\lambda_{3}^{\mathrm{aq}}-\lambda_{4}^{\mathrm{mic}}\right) /\left(\lambda_{3}-\lambda_{4}^{\mathrm{mic}}\right) \\
I_{4}=I_{3}^{\mathrm{aq}} F(C) k_{\mathrm{dif} 4} C\left(\lambda_{3}-\lambda_{4}^{\mathrm{mic}}\right)
\end{gathered}
$$

where $\lambda_{3}$ is given by eq 9 and $F(C)$ is the inhibition function $F(C)=1 /(1+k C)$, where $C$ is the micelle concentration (see eq 1$)$.

Using eqs 17 and 18 leads to an excellent fitting of the data (solid lines in Figure 3) with $k=0.50 \mathrm{M}^{-1}$. This latter value represents the inhibition constant of the SDS micelles. Dividing by $N_{\mathrm{ag}}=64$ gives the inhibition constant per SDS molecule at $0.01 \mathrm{M}^{-1}$. Considering that the SDS molecules are not homogeneously distributed, thus losing a large part of their ability to inhibit Ps formation in the homogeneously created positron spurs, this value is quite consistent with the low inhibition constant, $0.050 \mathrm{M}^{-1}$, found for methanesulfonate.

By contrast with the equations used for the reverse micelles, ${ }^{1}$ eq 18 does not include the possibility of direct Ps formation in the organic phase. This would introduce an additional term proportional to the micelle volume fraction and to the Ps formation probability in the micelles $\left(I_{4}^{\mathrm{mic}}\right)$. When the data are fitted on this basis, this latter parameter is found to be nil, whereas one would expect a value close to $38 \%$, corresponding 
to the $o$-Ps formation probability in dodecane. ${ }^{15}$ As was the case for the AOT/water system in isooctane, ${ }^{1}$ this reflects the much larger ability of water to slow the positrons as compared to the ability of alkanes, resulting in a nearly exclusive formation of Ps in the aqueous phase. On these grounds, the roughly linear increase of $I_{4}$ with $C$ shown in Figure 3 confirms the existence of the trapping process of Ps in the organic core of the micelles.

\section{Conclusion}

The present data confirm the ability of positron annihilation techniques to yield quantitative information on multicomponent systems. However, to obtain precise and reliable values of physical parameters descriptive of the systems studied, like the micelle core radius in the present case, the establishment of rigorous equations describing positronium diffusion is necessary. On this ground, it must be acknowledged that a better understanding of the properties and behavior of the probe, Ps, is still necessary. In this quest, studying known micellar systems of large dimensions should prove very fruitful to better define the diffusion properties of Ps.

Acknowledgment. Part of this work was carried out in the framework of the Programa de Cooperaçao Cientifica e Tecnica Luso-Francês (JNICT/French Embassy in Lisbon).

\section{References and Notes}

(1) Ferreira Marques, M. F.; Burrows, H. D.; Da Graça Miguel, M.; De Lima, A. P.; Lopes Gil, C.; Duplâtre G. J. Phys. Chem. 1996, 100, 7595.

(2) Fourth International Workshop on Positron and Positronium Chemistry, Journal de Physique. IV, Colloque, Le Mont Sainte-Odile, France, 1993; Billard, I., Ed.; Les Ulis: France, 1993; Vol. 3.
(3) Djermouni, B; Ache, H. J. J. Phys. Chem. 1979, 83, 2476.

(4) Alfassi, Z. B.; Ache, H. J. J. Phys. Chem. 1984, 88, 4347.

(5) Jean, Y. C.; Ache, H. J. J. Am. Chem. Soc. 1977, 99, 7509

(6) Jean, Y. C.; Ache, H. J. J. Am. Chem. Soc. 1978, 100, 984.

(7) Handel, E. D.; Ache, H. J. J. Chem. Phys. 1979, 71, 2083.

(8) Duplâtre, G.; Abbé, J. Ch.; Talamoni, J.; Machado, J. C.; Haessler, A. Chem. Phys. 1981, 57, 175 .

(9) Abbé, J. Ch.; Duplâtre, G; Maddock, A. G.; Talamoni, J; Haessler,

A. J. Inorg. Nucl. Chem. 1981, 43, 2603.

(10) Duplâtre, G.; Jonah, C. D. Radiat. Phys. Chem. 1985, 24, 557.

(11) Anbar, M.; Bambenek, M.; Ross, A. B. NSRDS-NBS 43, Part 1; National Bureau of Standards: Gaithersburg, MD, 1973.

(12) Ross, A. B. NSRDS-NBS 43, Supplement; National Bureau of Standards: Gaithersburg, MD, 1975.

(13) Buxton, G. V.; Greenstock, C. L.; Helman, W. P.; Ross, A. B. J. Phys. Chem. Ref. Data 1988, 17, 513.

(14) Didierjean, F.; Duplâtre, G.; Abbé, J. Ch. Radiat. Phys. Chem. 1991, 38,413 .

(15) Mogensen, O. E.; Jacobsen, F. M. Chem. Phys. 1982, 73, 223.

(16) Tao, S. J. J. Chem. Phys. 1970, 52, 752.

(17) Duplâtre, G. In Positron and Positronium Chemistry; Jean, Y. C.,

Ed.; World Scientific: Singapore, 1990; p 329.

(18) Waite, T. R. Phys. Rev. 1957, 107, 471.

(19) Peak, D.; Corbett, J. W. Phys. Rev. 1972, B5, 1226.

(20) Muller, N. In Micellization, Solubilization and Microemulsions;

Mittal, K. L., Ed.; Plenum Press: New York, 1977; Vol. 1, p 229.

(21) Lianos, P.; Zana, L. J. Colloid Interface Sci. 1981, 84, 100.

(22) Woolley, E. M.; Burchfield, T. E. J. Phys. Chem. 1984, 88, 2155.

(23) Doughty, D. A. J. Phys. Chem. 1981, 85, 3545.

(24) Mazer, N. A.; Benedek, G. B.; Carey, M. C. J. Phys. Chem. 1976, 80,1075 .

(25) Malliaris, A. Int. Rev. Phys. Chem. 1988, 7 (20), 95.

(26) Malliaris, A. Adv. Colloid Interface Sci. 1987, 27, 153.

(27) Tao, S. J. J. Chem. Phys. 1970, 52, 752.

(28) Fantola-Lazzarini, A. L.; Lazzarini, E. J. Inorg. Nucl. Chem. 1981, $43,2593$.

(29) Magalhaes, W. F.; Abbé, J. Ch.; Duplâtre, G. Struct. Chem. 1991, 2, 399 .

JP960644M 